\title{
El pensamiento computacional en educación. Análisis bibliométrico y temático
}

\section{Computational thinking in education. Bibliometric and thematic analysis}

\author{
Rosabel Roig-Vila \\ Universidad de Alicante. Alicante, España \\ rosabel.roig@ua.es \\ Víctor Moreno-Isac \\ Universidad de Alicante. Alicante, España \\ victor_moreno@hotmail.es
}

\begin{abstract}
RESUMEN
El pensamiento computacional se está considerando actualmente como una de las competencias más demandadas y, de ahí, su planteamiento en el contexto educativo. Este trabajo trata de analizar la literatura científica sobre la aplicación del pensamiento computacional en el ámbito educativo publicada en las colecciones principales de la base de datos Web of Science. Para lograrlo, se lleva a cabo una revisión sistemática donde se han tenido en cuenta las variables de año de publicación, los países con más producciones, las autorías más productivas en este campo y fuentes documentales con mayor número de publicaciones. Asimismo, se ha realizado una clasificación según los tipos de documentos y los métodos de investigación utilizados, así como las etapas educativas objeto de estudio y los lenguajes de programación utilizados. Se ha hallado una tendencia creciente de publicaciones en esta temática, donde España es uno de los países donde más se publica. Además, se ha observado cómo este campo de estudio se ha abordado desde los dos principales métodos de investigación -cuantitativo y cualitativo - y la etapa educativa más investigada es la educación primaria.
\end{abstract}

Palabras Clave: Pensamiento computacional, revisión sistemática, análisis de contenido, bibliometría, método de investigación, lenguaje de programación.

\begin{abstract}
The status of computational thinking as one of the most demanded skills explains why suggestions are currently being made to apply it within the educational context. This paper constitutes an attempt to analyze the scientific literature on the implementation of computational thinking in the field of education published in the most important Web of Science database collections. To achieve this, a systemic revision was made where attention was paid to four variables, namely: publication year; countries with more productions; the most productive authors in this field; and documentary sources with a higher number of publications. Added to this, we carried out a classification according to the types of documents and the research methods used, along with the educational stages under study and the programming language adopted. Publications are undoubtedly on an upward trend, Spain standing out as one of the most productive countries in this area. Likewise, evidence demonstrates not only that this field of study has been addressed using the two main research methods - quantitative and qualitative - but also that the emphasis has traditionally been placed on the primary education stage.
\end{abstract}

Keywords: Computational thinking, systematic review, data analysis, research method, programming language. 


\section{INTRODUCCIÓN}

Recientemente, la Organización para la Cooperación y el Desarrollo Económicos (OCDE), en su informe sobre creación de empleo y desarrollo económico local de 2018, augura que en España desaparecerán aproximadamente un $20 \%$ de empleos debido a la automatización (OCDE, 2018). Sin embargo, se da la paradoja de que, por un lado, muchos de estos países presentan altos índices de paro, mientras que, por otro lado, se quedan muchos puestos de trabajo sin cubrir. Zapata-Ros (2015) afirma que multitud de estos puestos tienen una relación directa con el desarrollo del pensamiento computacional.

Es una realidad que, actualmente, el pensamiento computacional está adquiriendo cada vez más relevancia por el rápido desarrollo de las nuevas tecnologías (Denning, 2017). Esto está dando lugar a la creación de una tendencia global que empieza a considerar la enseñanza de la programación en el aula como una actividad fundamental de cara al futuro (Naughton, 2012). Se trata de poder desarrollar las competencias fundamentales vinculadas a la realidad del mundo laboral y personal al que se enfrenta el alumnado (Gutiérrez y Serrano, 2016; Sancho y Padilla, 2016; Vázquez-Cano y Ferrer, 2015). Ello ha provocado que muchos expertos coincidan en un hecho: la sociedad, los sistemas de producción, de servicios y de consumo demandan profesionales más cualificados y cualificadas en las industrias de la información (Zapata-Ros, 2015).

Por otro lado, Adell, Llopis, Esteve y Valedeolivas (2019) declaran, que resulta cuestionable el hecho de que todos los profesionales y las profesionales actuales o de un futuro que vayan a realizar su labor con ordenadores o cualquier tecnología de procesamiento de la información, tengan la necesidad de saber diseñar algoritmos o programas más allá de modificar determinadas funcionalidades básicas de un programa. Esto se justifica con el argumento de que no todas las profesiones se enfrentarán a un tipo de problemas solucionables mediante el pensamiento computacional. Sea como sea, el mundo de la computación nos rodea y, a su vez, los avances tecnológicos de la revolución digital nos sitúan ante lo que algunos autores y autoras vienen a denominar la cuarta revolución industrial (Cabrera, 2017).

Ante ello, nos encontramos con la necesidad de una reforma profunda de los actuales sistemas educativos (Valverde, Fernández y Garrido, 2015). Se continúa privilegiando la enseñanza del contenido sobre el desarrollo de destrezas y habilidades cognitivas que permite el desarrollo de las estudiantes y los estudiantes (Balladares, Avilés y Pérez, 2016), y ello debe cambiarse. Las soluciones podrían pasar por la incorporación de nuevas metodologías en el proceso de enseñanza aprendizaje y modificar sustancialmente el modelo organizativo de las instituciones educativas (Valverde et al., 2015).

En este planteamiento tienen cabida experiencias educativas basadas en el pensamiento computacional. Por un lado, el desarrollo del pensamiento computacional en infantes y jóvenes hace que "puedan pensar de manera diferente, expresarse a través de una variedad de medios, resolver problemas del mundo real y analizar temas cotidianos desde una perspectiva diferente" (Bocconi, Chioccariello, Dettori, Ferrari y Engelhardt, 2016, p. 25). Por otro lado, puede servir para poder impulsar el crecimiento económico, cubrir puestos de trabajo TIC y prepararse para futuros empleos (Bocconi et al., 2016; Bocconi, Chioccariello, Dettori, Ferrari, Engelhardt, Kampylis, Punie, 2016).

El pensamiento computacional en educación. Análisis bibliométrico y temático. Roig-Vila, R. y Moreno, V.

Página 1 de 24 


\section{Delimitando el concepto de pensamiento computacional}

La mayoría de las publicaciones referentes al pensamiento computacional atribuyen el origen del concepto a Wing (2006), según la cual el pensamiento computacional:

Implica resolver problemas, diseñar sistemas y comprender el comportamiento humano, basándose en los conceptos fundamentales de la ciencia de la computación. El pensamiento computacional incluye una amplia variedad de herramientas mentales que reflejan la amplitud del campo de la computación. Además representa una actitud y unas habilidades universales que todos los individuos deberían aprender a usar (2006, p.33).

Asimismo, Wing (2006) también lo catalogó como un elemento fundamental para vivir en el siglo XXI, y llegó a equipararlo con las matemáticas y la escritura. Años después, trató de aclarar las implicaciones que posee el término cuando afirmó que:

El pensamiento computacional es un enfoque para la solución de problemas, construcción de sistemas y la comprensión del comportamiento humano, que se basa en el poder y los límites de la computación. El pensamiento computacional ya ha comenzado a influir en muchas disciplinas. De cara al futuro, podemos anticipar incluso efectos más profundos del pensamiento computacional en la ciencia, la tecnología y la sociedad (Wing, 2009, p.1).

En adición a lo aportado por Wing, la Computer Science Teacher Association (CSTA) y la International Society in Technology in Education (ISTE) trataron de definirlo en 2011 como "un enfoque para resolver un determinado problema que empodera la integración de tecnologías digitales con ideas humanas. No reemplaza el énfasis en creatividad, razonamiento o pensamiento crítico, pero refuerza esas habilidades al tiempo que realza formas de organizar el problema que el computador pueda ayudar" (CSTA y ISTE, 2011). En definitiva, se trata de una forma de pensar propicia para el análisis y la relación de ideas, para la organización y la representación lógica (Zapata-Ros, 2015).

Por otro lado, Valverde et al. (2015) entienden el pensamiento computacional como un concepto complejo en sí mismo, ya que permite que se le relacione con una competencia completa, de un grado de complejidad alto que, al mismo tiempo, se puede relacionar con niveles de pensamiento abstracto, matemático y pragmático aplicados en diferentes momentos de nuestra vida cotidiana. Además, este tipo de pensamiento no es sinónimo únicamente de programación de un ordenador, ya que permite ser desarrollado sin el uso de un ordenador, únicamente utilizando papel y lápiz (Balladares et al., 2016).

A esta afirmación se le puede sumar lo afirmado por Pérez y Roig-Vila (2015), al indicar que el pensamiento computacional está relacionado con otros tipos de pensamiento, como el matemático, lógico y crítico, dado que comparten habilidades cognitivas comunes tales como el reconocimiento de patrones, abstracción y modelación. Además, indican que la finalidad del pensamiento computacional es que, a partir del reconocimiento de los problemas reales de las actividades diarias, se propongan soluciones con la aplicación de herramientas informáticas. A ello se añade la consideración de atribuir al pensamiento computacional un alto nivel de abstracción y un enfoque algorítmico para resolver cualquier tipo de problema (García-Peñalvo, 2016; Pinto-Llorente, Casillas-Martín, Cabezas y García-Peñalvo, 2018).

El pensamiento computacional en educación. Análisis bibliométrico y temático. Roig-Vila, R. y Moreno, V. 
Asimismo, el pensamiento computacional también se puede considerar como una metodología que se basa en tratar de implementar algunos de los conceptos básicos de las ciencias de la computación para tratar de resolver problemas cotidianos, diseñar sistemas domésticos y realizar tareas rutinarias. Esto permite abordar los problemas de una forma novedosa para poder resolver con eficacia y satisfactoriamente problemas que, de otro modo, no son tratables por una persona (Basogain, Olabe y Olabe, 2015).

Conviene destacar, asimismo, la propuesta llevada a cabo por Brennan y Resnick (2012) en torno a las tres dimensiones del pensamiento computacional:

- Conceptos computacionales: utilizados por programadores para la elaboración de programas aplicables en cualquier ámbito (secuencias, ciclos, paralelismos, eventos, condicionales, operadores y datos).

- Prácticas computacionales: las que se utilizan cuando se programan y se centran en el cómo se está aprendiendo y no en el qué (iteración, ensayo y depuración, abstracción y modularidad).

- Perspectivas computacionales: refiriéndose a las que los usuarios y usuarias construyen sobre sí mismos y su entorno gracias a la programación (preguntar, conectar, expresar).

Por último, Repenning, Basawaptna y Escherle (2017) identifican tres etapas del proceso que el pensamiento computacional debe suscitar: formulación de un problema (abstracción), expresión de la solución (automatización) y ejecución y evaluación (análisis).

Todas estas consideraciones deben hacernos reflexionar sobre la necesidad de lograr un mayor consenso al delimitar el concepto de pensamiento computacional. Esto facilitaría saber dónde se sitúa el punto exacto desde el cual partir, con el fin de desarrollar los instrumentos que faciliten su incorporación al contexto educativo como una competencia fundamental para poder desenvolvernos en la sociedad actual.

\section{El pensamiento computacional en educación}

Cuando se aborda de qué manera incluir el pensamiento computacional en la escuela, surge la necesidad de aclarar que el pensamiento computacional no se limita a la programación o el diseño e implementación de un programa informático (Olabe, Basogain, Olabe, Maíz y Castaño, 2014). El pensamiento computacional amplía las facultades con la ayuda de las herramientas informáticas, donde la imaginación y la creatividad juegan un papel fundamental en las nuevas ideas de los mundos virtuales (Balladares et al. , 2016).

Por otro lado, suele ocurrir que una de las primeras ideas que surgen cuando se aborda el concepto de pensamiento computacional, es que se tiene la creencia de que se trata de una competencia exclusiva a desarrollar en el ámbito de la ingeniería informática y la computación, cuando no es así (Basogain et al., 2015). Numerosos juegos y proyectos abarcan una nueva iniciativa educativa, basada en novedosas modalidades del juego mismo (Del-Moral, Guzmán y Fernández, 2018; Wartenweiler, 2018), que aboga firmemente por la incorporación del pensamiento computacional, la programación informática y la robótica en los colegios. Valverde et al. (2015) consideran que el

El pensamiento computacional en educación. Análisis bibliométrico y temático. Roig-Vila, R. y Moreno, V. 
pensamiento computacional es una competencia compleja, y que está relacionada con un modelo de conceptualización específica de los seres humanos a la hora de desarrollar ideas. En efecto, está conectado al pensamiento abstracto-matemático y al pragmático ingenieril, pero se aplica en múltiples aspectos de nuestra vida diaria. No debe ser, por tanto, un sinónimo de capacidad de programación de un ordenador, ya que requiere un pensamiento en diferentes niveles de abstracción, y no tiene por qué estar conectado a los dispositivos electrónicos.

Respecto a los inicios de la inclusión de una competencia como el pensamiento computacional en educación, cabe destacar que fue Papert (1980) el primero en enlazar una corriente clásica de aprendizaje que se apoyaba en la tecnología. Se trataba de una alternativa en la forma de enfocar la enseñanza del pensamiento computacional. Blinckstein (2013), citado por Zapata-Ros (2015), considera que Papert ha estado en el centro de tres revoluciones: el desarrollo del pensamiento en la infancia, la inteligencia artificial, y las tecnologías informáticas para la educación. La visión de Papert se suele resumir con la frase: "son los niños los que deben educar a los ordenadores, no los ordenadores lo que tienen que educar a los niños"(Zapata-Ros, 2015, p.3). Esta afirmación debe ser tomada como punto de partida si queremos alcanzar un desarrollo de la competencia que se centre en las capacidades del alumnado (García-López, Gutiérrez, Pastor y Romo, 2018) y no únicamente en su dominio de determinados lenguajes de programación.

Sin embargo, toda esta controversia y falta de acuerdo da pie a un debate sobre cómo debe incorporarse el pensamiento computacional en el currículo educativo. Una opción evidente es incluir una asignatura independiente en la educación obligatoria (Bocconi et al.,2016). Sin embargo, son muchos los que defienden la transversalidad, entre ellas la propia Wing (2006), integrando los diferentes elementos clave del pensamiento computacional en diversas áreas y asignaturas (Adell et al., 2019). Podemos encontrar aportaciones muy interesantes en este campo, pero la realidad es que aún no se ha desarrollado una didáctica específica sobre el pensamiento computacional, y cómo abordar la formación, tanto inicial como permanente, del profesorado, quizás por tratarse de un tema sin apenas recorrido (Adell et al., 2019; González, Estebanell y Perascaula, 2018; Román-González, 2015; Yadav, Hong y Stephenson, 2016; Yadav, Mayfield, Zhou, Hambusch y Korb, 2014).

Además, tampoco existen muchas propuestas relacionadas con la evaluación del aprendizaje del pensamiento computacional, por lo que es difícil saber en qué grado se ha desarrollado esa habilidad de pensamiento (Grover y Pea, 2013). En el mismo sentido, Zapata-Ros (2015) señala que, aunque los efectos positivos pueden ser observados desde la práctica y la experiencia, sigue existiendo esa necesidad de elaborar instrumentos para poder realizar una medición del pensamiento computacional. Todo esto ha propiciado el desarrollo de investigaciones en torno a la evaluación del pensamiento computacional, tales como las propuestas de Yagci (2019), Kong, Chiu y Lai (2018) y Ioannou y Makridou (2018).

Por todo lo expuesto, y debido a la relevancia que va a adquirir el desarrollo del pensamiento computacional en el ámbito educativo, nos planteamos como objetivo llevar a cabo un análisis bibliométrico de toda la literatura científica indexada y publicada en la

El pensamiento computacional en educación. Análisis bibliométrico y temático. Roig-Vila, R. y Moreno, V. 
base de datos Web of Science sobre la investigación educativa referida al pensamiento computacional hasta el año 2018. De forma más específica, nos proponemos realizar dicho análisis a partir de las siguientes variables: la evolución temporal de las producciones, realizando una comparación entre la producción en investigación educativa y la producción general, países con mayor número de producciones, revistas más destacadas en la difusión del pensamiento computacional en educación, autoras y autores más productivos, grado de colaboración entre investigadoras e investigadores, tipos de documentos publicados y metodologías de investigación utilizadas, poblaciones empleadas para las muestras y lenguajes de programación o plataformas más utilizados para implementar el pensamiento computacional en el contexto educativo.

\section{MÉTODO}

Hemos realizado una revisión sistemática de la literatura (en inglés systematic literature review (SLR) (Brereton, Kitchenham, Budgen, Turner y Khalil, 2007) a partir de los estándares PRISMA referidos al diseño metodológico, a saber, protocolo, proceso de búsqueda, selección y síntesis de resultados (Moher, Liberati, Tetzlaff, Altman, \& The PRISMA group, 2009). Con ello, identificamos, interpretamos y evaluamos los datos disponibles en un periodo de tiempo referidos a un campo de investigación concreto (Centre for Reviews and Dissemination, 2009; Higgins y Green, 2006; Kitchenham, 2004), en este caso, el pensamiento computacional en el ámbito educativo.

\section{Muestra}

La unidad de análisis de esta revisión se ha compuesto de 189 documentos, publicados en las colecciones principales de la base de datos Web Of Science. El total de la muestra seleccionada para esta revisión referida a los diversos artículos han sido leídos en su totalidad, con mayor o menor profundidad. El periodo de tiempo seleccionado abarca desde el primer artículo publicado sobre este tema en el año 2008 hasta 2018 inclusive. Cabe destacar que la muestra se encuentra repartida entre artículos académicos y proceeding papers.

Para llevar a cabo la búsqueda de la unidad de análisis se ha utilizado la base de datos Web Of Science (WoS). Concretamente se ha seleccionado la colección principal, que está compuesta por: Citation Index Expanded (SCI- EXPANDED), Social Science Citation Index (SSCI), Arts and Humanities Citation Index (A-HCI), Conference Proceedings Citation Index-Science (CPIC-S), Conference Proceedings Citation IndexSocial Science and Humanities (CPCI-SSH), Book Citation Index-Science (BKCI-S), Book Citation Index-Social Science and Humanities (BKCI-SSH). En esta serie de bases de datos se encuentran recogidas las principales revistas catalogadas con mayor factor de impacto en el ámbito de las ciencias y las ciencias sociales, disponibles en la base de datos WoS.

\section{Procedimiento}

Para realizar la búsqueda, se utilizó la estrategia de utilizar el término "computational thinking" en el buscador de las colecciones principales de WoS, con el fin de localizar los documentos que mencionaran dichos términos en el título. En una primera búsqueda se obtuvo un total de 584 documentos. De estos 584 documentos se realizó una gráfica

El pensamiento computacional en educación. Análisis bibliométrico y temático. Roig-Vila, R. y Moreno, V. 
de fechas para comparar la evolución de la investigación sobre pensamiento computacional en el ámbito científico en general y, así, poder compararla con la evolución del pensamiento computacional en el ámbito de la investigación educativa. Seguidamente, se refinó la búsqueda, seleccionando el apartado de investigación educativa, dentro de las categorías de WoS. Por último, se excluyeron los documentos del año 2019, por no ser un año finalizado. Aplicando esta estrategia, obtuvimos un total de 213 documentos datados entre los años 2008 y 2018.

A continuación, con el fin de seleccionar la muestra definitiva, se procedió a la lectura de todos los resúmenes y de los textos completos, con mayor o menor detalle, en base a poder extraer la información que se quería obtener. El objeto de esta lectura era tener la certeza de que todos los documentos investigaban elementos relacionados con el pensamiento computacional en el ámbito educativo, ya fuera a través de experiencias empíricas o análisis teóricos. Además, se empleó como criterio de exclusión aquellos artículos que no permitieran, como mínimo, el acceso al resumen. Igualmente, tampoco se seleccionaron los documentos que no abordaran de alguna manera el pensamiento computacional desde una perspectiva educativa. Tras realizar una rigurosa lectura, se seleccionaron un total de 189 artículos.

En la lectura de los artículos se procedió a realizar la selección de datos necesarios para llevar a cabo la presente investigación. Para ello se atendió a las siguientes variables: año y país de publicación, fuente documental en la que se ha publicado, autor, autora o autores del documento, características de los participantes y las participantes del estudio, tipo de documento, metodología de investigación utilizada y plataforma o lenguaje de programación con la que se ha trabajado el pensamiento computacional, si lo hubiera. Respecto a la metodología investigadora utilizada, primero se diferenció entre un enfoque cualitativo, cuantitativo o mixto y, seguidamente, se clasificó según el tipo de investigación realizado.

\section{Diseño y análisis de resultados}

La siguiente investigación se considera un estudio ex post facto retrospectivo según la clasificación realizada por Montero y León (2007), y se basa en los planes de trabajo planteados en otros estudios bibliométricos anteriores (García-Fernández, Inglés, Gonzálvez, Vicent y Gómez, 2013; García-Fernández et al., 2016). Para analizar las diferentes variables se han realizado análisis descriptivos y de frecuencias. Los análisis y cálculos estadísticos se han sido desarrollado mediante el programa estadístico SPSS 25.

\section{RESULTADOS}

\section{Producción temporal}

Para el análisis cronológico de publicaciones, se ha realizado una comparativa entre lo publicado en relación con el pensamiento computacional en todos los ámbitos de investigación y lo publicado en el ámbito de la investigación educativa. En la fig. 1 de publicaciones generales, se puede observar cómo la primera publicación aparece en el año 1979, mientras que en la fig. 2 se observa que no es hasta 2008 cuando aparece una publicación sobre pensamiento computacional desde una perspectiva de investigación

El pensamiento computacional en educación. Análisis bibliométrico y temático. Roig-Vila, R. y Moreno, V. 
educativa. Asimismo, se puede observar el continuo crecimiento que ha habido de la investigación en este campo durante estos últimos años, tanto a nivel general, es decir, en todos los ámbitos de investigación, como, de forma específica, en el ámbito educativo. Así, encontramos que el periodo más productivo en ambos casos se comprende entre los años 2013 y 2018: las publicaciones de estos años suponen el 83,56\% de la producción total a nivel general; y la mayor producción se dio en el año 2017 con un total de 149 publicaciones.

En cuanto al ámbito de la investigación educativa, en los primeros años (2008-2012) se publican anualmente entre una y cinco investigaciones, y no es hasta el año 2013 cuando encontramos el primer repunte, con un total de 13 publicaciones, alcanzando el mayor número en el año 2017 con 69 publicaciones, lo que supone que la investigación educativa abarcó el 46,3\% del total investigado en este año. Como ocurriera en el caso de todos los ámbitos de investigación, en el periodo entre 2013 y 2018 se recoge el mayor número de publicaciones, pero, en este caso, las publicaciones existentes entre estos años suponen el $93,42 \%$ del total.

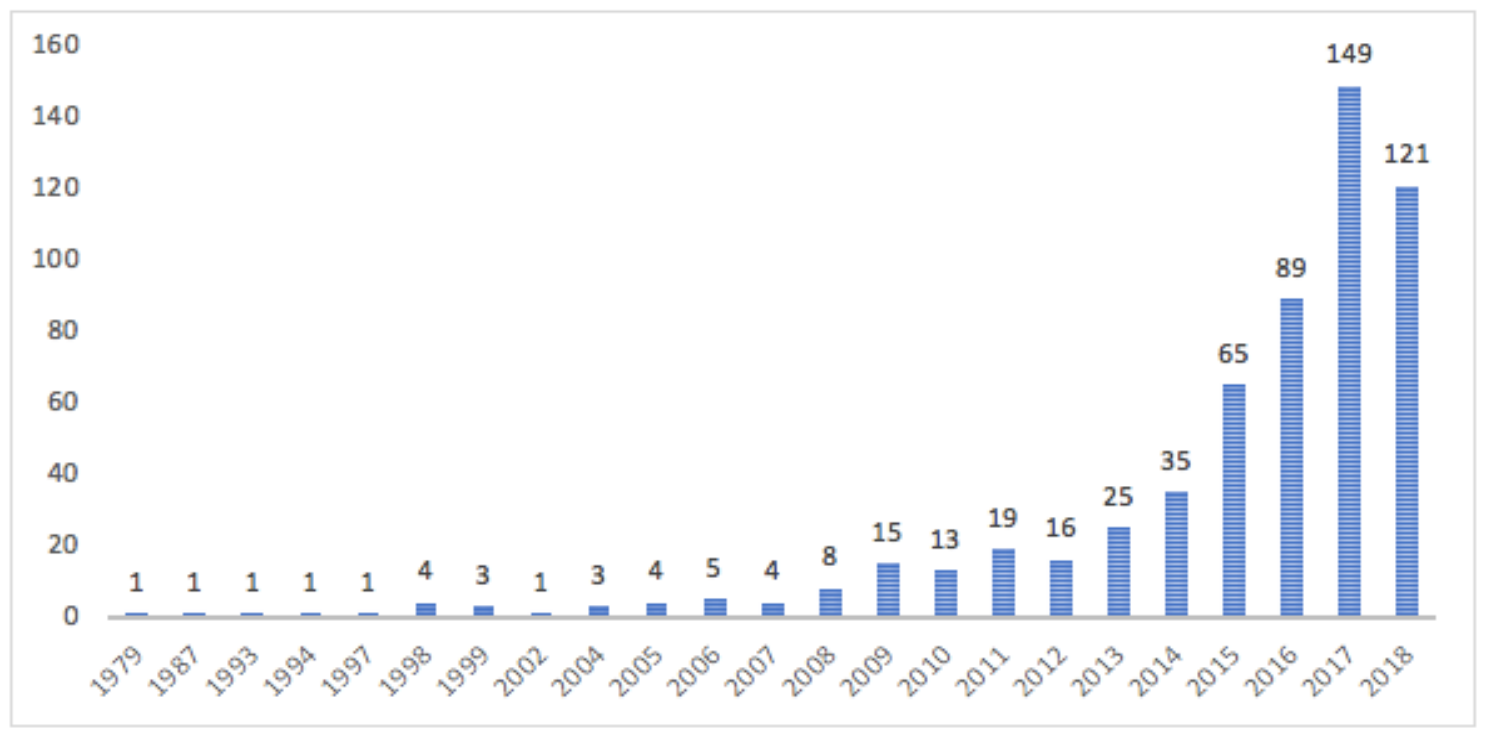

Fig. 1. Distribución anual del número de publicaciones en todos los ámbitos de investigación.

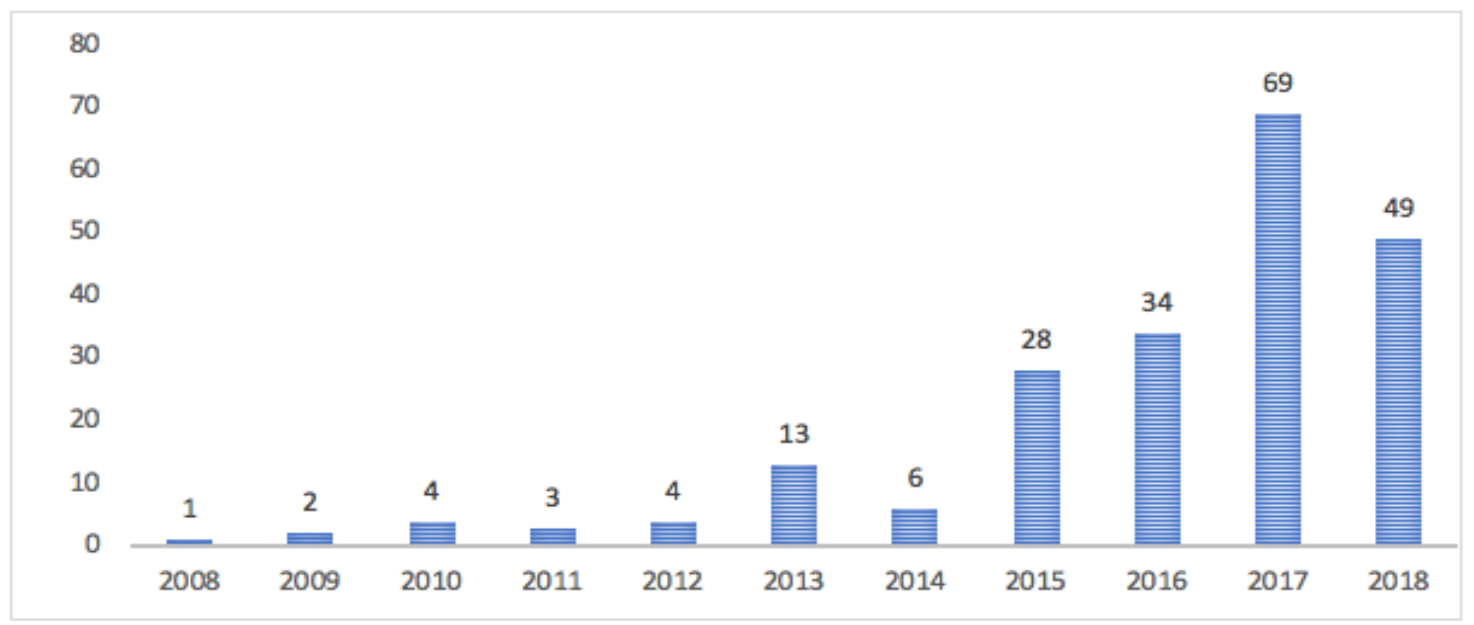

El pensamiento computacional en educación. Análisis bibliométrico y temático. Roig-Vila, R. y Moreno, V. 
Fig. 2. Distribución anual del número de publicaciones en el el ámbito de la investigación educativa.

\section{Producción por zonas geográficas}

En lo que se refiere a la distribución geográfica de las respectivas publicaciones, en la tabla 1 se muestra la relación de países con el total de publicaciones realizadas. En este caso, Estados Unidos cuenta con el mayor número (66 publicaciones). Los países que le siguen son: China (36), España (21) y Canadá e Inglaterra (8).

Tabla 1

Distribución geográfica de las publicaciones

\begin{tabular}{lclc}
\hline País & Publicaciones & País & Publicaciones \\
\hline Estados Unidos & 66 & China & 36 \\
España & 21 & Canadá & 8 \\
Inglaterra & 8 & Alemania & 7 \\
Italia & 6 & Brasil & 5 \\
Singapur & 5 & Australia & 4 \\
Turquía & 4 & Dinamarca & 4 \\
Lituania & 4 & Holanda & 4 \\
Corea del sur & 4 & Colombia & 3 \\
Chipre & 3 & Grecia & 2 \\
India & 3 & Ecuador & 2 \\
Japón & 2 & Nueva Zelanda & 2 \\
Portugal & 2 & Sudáfrica & 2 \\
Escocia & 2 & Taiwán & 2 \\
Austria & 2 & Bélgica & 1 \\
Croacia & 1 & Argentina & 1 \\
República Dominicana & 1 & Estonia & 1 \\
Finlandia & 1 & Francia & 1 \\
Israel & 1 & Noruega & 1 \\
Perú & 1 & Méjico & 1 \\
Suiza & & Irlanda & 1 \\
\hline & & & 2 \\
\hline
\end{tabular}

El pensamiento computacional en educación. Análisis bibliométrico y temático. Roig-Vila, R. y Moreno, V. 


\section{Producción por fuentes o revistas}

Respecto a las fuentes de los respectivos documentos, cabe distinguir, en primer lugar, los tipos de documentos: del total de los 189 seleccionados para este estudio, 104 son artículos de revistas y 85 proceeding papers. Eso quiere decir que los artículos representan un $55,02 \%$ del total de la muestra, mientras que los proceeding papers suponen un $44,98 \%$.

Por otro lado, profundizando en las fuentes, los documentos que conforman el total de la muestra han sido publicados en un total de 116 fuentes distintas, distribuidos entre recopilatorios de congresos, revistas y un libro. La tabla 2 incluye un listado de los títulos con más publicaciones -a partir de cinco- en relación con la investigación educativa sobre el pensamiento computacional. Las dos fuentes con más publicaciones son el libro titulado Emerging research practice and policy on computational thinking (19) y el libro de actas del congreso INTED de 2017 (INTED proceedings) (9).

Tabla 2

Fuentes documentales con mayor volumen de publicaciones, país de procedencia y total de publicaciones.

\begin{tabular}{llc}
\hline Fuente & País & Frecuencia \\
\hline $\begin{array}{l}\text { Emerging Research Practice and Policy on } \\
\quad \text { Computational Thinking }\end{array}$ & Alemania & 19 \\
$\begin{array}{l}\text { Inted Proceedings } \\
\text { 26th International on Computers in Education }\end{array}$ & España & 9 \\
$\quad$ ICCE 2018 & Filipinas & 7 \\
ASEE Annual Conference Exposition & Estados Unidos & 7 \\
Edulearn Proceedings & España & 7 \\
Frontiers in Education Conference & Estados Unidos & 7 \\
$\begin{array}{l}\text { Informatics in Education } \\
\text { Red Revista de Educación a Distancia }\end{array}$ & Lituania & 7 \\
Computers and Education & España & 7 \\
Education in the Knowledge Society & Reino Unido & 6 \\
Proceedings of the European Conference & España & 6 \\
$\quad$ On Game Based Learning & Alemania & 6 \\
TechTrends & Estados Unidos & 6 \\
Advances in intelligent System Research & Francia & 5 \\
\hline
\end{tabular}

El pensamiento computacional en educación. Análisis bibliométrico y temático. Roig-Vila, R. y Moreno, V. 


\section{Producción por autores}

En relación con las firmas de los documentos, se han identificado un total de 511 autoras y autores. En la figura 3 se observa la clasificación de autores, aplicando la tipificación de dichos autores y autoras en base al modelo de Crane (1969), el cual establece una clasificación a partir de la producción de una autoras o un autor sobre un tema determinado y distingue, así, entre transeúntes (1 única publicación), aspirantes (de 2 a 4 publicaciones), productores moderados (de 5 a 9 publicaciones) y grandes productores (más de 10 producciones). Partiendo de esta clasificación, se ha constatado que una gran mayoría de los autores y autoras que han publicado sobre el pensamiento computacional en investigación educativa son transeúntes, ya que representan un $86,9 \%$ del total, en comparación con el resto. Además, el 12,52\% son aspirantes y, únicamente un 0,58\% del total son productores o productoras moderadas, y no se observa ningún gran productor o productora en este campo. Asimismo, en la tabla 3 se muestran los autores y autoras catalogados como productoras o productores moderados y algunos aspirantes con 4 o 3 publicaciones respectivamente: únicamente encontramos tres productores moderados, a saber, los autores Song-Charg Kong y Aman Yadav con 6 trabajos cada uno, y Uri j. Wilenski, con 5.

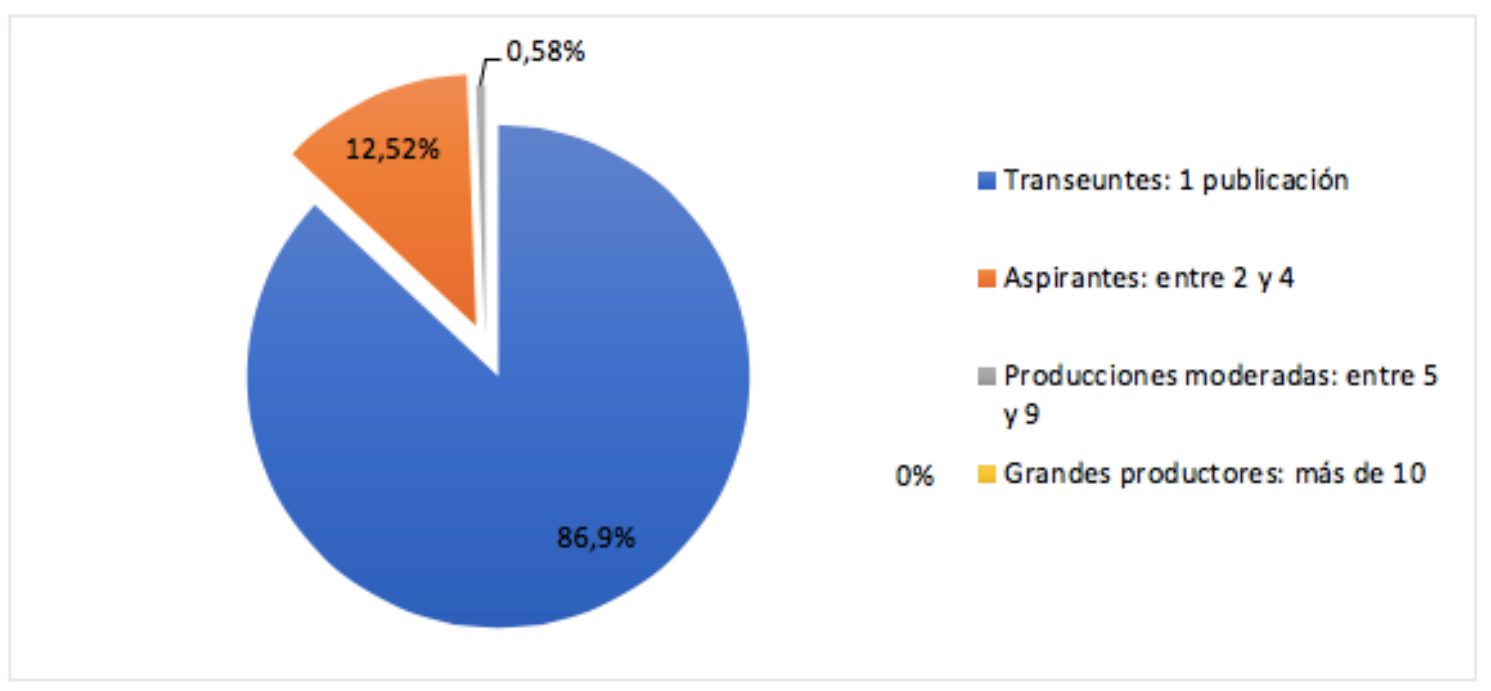

Fig. 3. Clasificación de autores según producción

Tabla 3

Principales autoras y autores clasificados como productoras o productores moderados $o$ aspirantes, número de artículos firmados y su filiación institucional

\begin{tabular}{lll}
\hline \multirow{2}{*}{ Autores } & Filiación & Frecuencia \\
& Institucional & \\
\hline
\end{tabular}

El pensamiento computacional en educación. Análisis bibliométrico y temático. Roig-Vila, R. y Moreno, V. 


\begin{tabular}{|c|c|c|}
\hline Song-Charg Kong & $\begin{array}{l}\text { Iowa State University, } \\
\text { Estados Unidos }\end{array}$ & 6 \\
\hline Aman Yadav & $\begin{array}{l}\text { Michigan State University, } \\
\text { Estados Unidos }\end{array}$ & 6 \\
\hline Uri J. Wilensky & $\begin{array}{l}\text { North-western University, } \\
\text { Estados Unidos }\end{array}$ & 5 \\
\hline Jon Good & $\begin{array}{l}\text { Oakland Schools, } \\
\text { Estados Unidos }\end{array}$ & 4 \\
\hline Suchi Grover & $\begin{array}{l}\text { Edifinity University, } \\
\text { Singapur }\end{array}$ & 4 \\
\hline Valentina Dagiene & $\begin{array}{l}\text { Vilnius University, } \\
\text { Lituania }\end{array}$ & 3 \\
\hline Chee-Kit Looi & $\begin{array}{l}\text { Nanyang Technology University, } \\
\text { Singapur }\end{array}$ & 3 \\
\hline Korbinian Moeller & $\begin{array}{l}\text { University of Tubingen, } \\
\text { Alemania }\end{array}$ & 3 \\
\hline Manuel Ninaus & $\begin{array}{l}\text { University of Tubingen, } \\
\text { Alemania }\end{array}$ & 3 \\
\hline Joke Voogt & $\begin{array}{l}\text { Windesheim University of Applied } \\
\text { Sciences, } \\
\text { Holanda }\end{array}$ & 3 \\
\hline Lei Wang & $\begin{array}{l}\text { Northeast normal University, } \\
\text { China }\end{array}$ & 3 \\
\hline David Weintrop & $\begin{array}{l}\text { Maryland University, } \\
\text { Estados Unidos }\end{array}$ & 3 \\
\hline
\end{tabular}

Por otro lado, respecto al número de firmas de los documentos seleccionados para el análisis, podemos observar en la tabla 4 cómo un $84,13 \%$ son trabajos escritos en coautoría, y solamente el $15,87 \%$ es de un único autor o autora. Además, los trabajos en coautoría realizados por 4 o más autores o autoras son los más frecuentes, representando un $34,92 \%$ del total de las publicaciones.

Tabla 4

Distribución de los documentos en base al número de autores firmantes

El pensamiento computacional en educación. Análisis bibliométrico y temático. Roig-Vila, R. y Moreno, V. 


\begin{tabular}{lcc}
\hline Número de firmas por documento & Frecuencia & Porcentaje \\
\hline 1 & 30 & 15,87 \\
2 & 49 & 25,93 \\
3 & 44 & 23,28 \\
4 o más & 66 & 34,92 \\
Total & 189 & 100 \\
\hline
\end{tabular}

\section{Tipos de documentos y metodologías utilizadas}

Respecto a los tipos de documentos que se han encontrado en la búsqueda, son varios los aspectos a distinguir, los cuales quedan esquematizados en la tabla 5. En primer lugar, se ha realizado una separación entre los documentos de carácter teórico, los cuales engloban las revisiones y ensayos teóricos, y los documentos que recogen experiencias de carácter empírico. Una vez llevada a cabo la clasificación, se observó que, de los 189 documentos, 66 de ellos tenían un carácter exclusivamente teórico (34.92\%), mientras que 123 $(65,08 \%)$ recogían experiencias empíricas de diversos tipos.

Seguidamente, se lleva a cabo una clasificación según la tipología de documentos teóricos. Hemos apreciado dos grandes grupos: ensayos exclusivamente teóricos y revisiones. Así, de los 66 documentos teóricos analizados, 50 de ellos son ensayos teóricos $(75,25 \%)$ y los 16 restantes corresponden a revisiones de diferentes aspectos sobre el pensamiento computacional y la educación $(24,75 \%)$.

De igual forma se procede a realizar una clasificación de las experiencias empíricas, las cuales suponen un total de 123 documentos. Se clasificaron según el método de investigación utilizado o, en su caso, experiencias de aprendizaje. Cabe destacar que varios de estos artículos trataban de realizar una validación de un instrumento de evaluación aplicable al pensamiento computacional. Este tipo de documento también ha sido considerado experiencia empírica, debido a que el proceso de validación presenta muchas similitudes con las investigaciones de carácter cuantitativo. Por consiguiente, de los 123 documentos que reflejan experiencias empíricas, 81 de ellos corresponden a investigaciones, mientras que 42 relatan diferentes experiencias de aprendizaje.

En cuanto a las investigaciones, 34 utilizan un método cuantitativo de investigación, 23 un método cualitativo y 13 un método mixto. Además, 9 de ellos tratan de validar un instrumento y 42 narran experiencias de aprendizaje.

Respecto al tipo de investigación cuantitativa realizada, se han tenido en cuenta los procedimientos utilizados en las investigaciones mixtas y las puramente experimentales. El total de documentos que presenta investigaciones de este tipo es de 49, donde 12 de ellas son experimentales $(24,49 \%), 24$ son cuasi experimentales $(48,98 \%), 7$ son correlacionales $(14,28 \%)$ y 6 son descriptivos $(12,25 \%)$.

El pensamiento computacional en educación. Análisis bibliométrico y temático. Roig-Vila, R. y Moreno, V. 
Por último, en lo referente a las investigaciones cualitativas, se tomó en consideración el tipo de método empleado para la recogida de datos. Como ocurriera con las investigaciones cuantitativas, se ha tenido en consideración también la parte cualitativa de las investigaciones mixtas. Esto hace que el total de investigaciones cualitativas que se analizaron fuera de 36. En ellas se observó que 21 utilizaban cuestionarios de respuesta abierta para la recogida de datos; 5 utilizaban entrevistas semiestructuradas donde el investigador grababa las conversaciones; 4 realizaban una observación directa sin intervenir en el proceso; otras 4 planteaban pequeños grupos de discusión y 2 habían realizado estudios de casos muy concretos de uno o dos participantes respectivamente.

Tabla 5

Distribución de los documentos según los tipos de documentos, documentos teóricos, documentos empíricos, métodos cuantitativos y métodos cualitativos

\begin{tabular}{lcc}
\hline Tipo de documento & Frecuencia & Porcentaje \\
\hline Teóricos & 66 & 34,92 \\
Empíricos & 123 & 65,08 \\
Total & 189 & 100 \\
& Frecuencia & Porcentaje \\
Documentos teóricos & 50 & 75,25 \\
\hline Artículos teóricos & 16 & 24,75 \\
Revisiones & 66 & 100 \\
Total & & Porcentaje \\
& Frecuencia & 34,15 \\
Documentos empíricos & 42 & 29,27 \\
\hline Experiencias de aprendizaje & 36 & 18,70 \\
Investigaciones cuantitativas & 23 & 10,56 \\
Investigaciones cualitativas & 13 & 7,32 \\
Investigaciones mixtas & 9 & 100 \\
Validación de instrumentos de & 123 & Porcentaje \\
evaluación & & 24,49 \\
Total & Frecuencia & 48,98 \\
Investigaciones & 12 & 14,28 \\
cuantitativas y mixtas & & \\
\hline Experimental & & \\
Cuasi-experimental & & \\
Correlacional & & \\
\hline & & \\
\hline
\end{tabular}

El pensamiento computacional en educación. Análisis bibliométrico y temático. Roig-Vila, R. y Moreno, V. 


\begin{tabular}{lcc}
\hline Descriptivo & 6 & 12,25 \\
Total & 49 & 100 \\
& & \\
Investigaciones \\
cualitativas y mixtas & Frecuencia & Porcentaje \\
\hline Cuestionarios & 21 & 58,33 \\
Entrevistas & 5 & 13,89 \\
Observación directa & 4 & 11,11 \\
Grupos de discusión & 4 & 11,11 \\
Estudio de caso & 2 & 5,56 \\
Total & 36 & 100 \\
\hline
\end{tabular}

\section{Población de referencia}

Respecto a las muestras utilizadas o, en su caso, a los grupos a los que iba dirigido el contenido de los respectivos documentos, se llevó a cabo una clasificación teniendo en cuenta la etapa educativa hacia la que iba dirigido el tema central del documento. Cabe decir que se ha tenido en cuenta la distribución de etapas del sistema educativo español y que se ha diferenciado la formación inicial del profesorado, de la etapa universitaria en general, al tratarse de formación específica. Además, muchos de los documentos, especialmente los de carácter teórico, no hacían referencia a ninguna edad en concreto. Así, como muestra la tabla 6, en 56 de los 189 artículos no figura una población de referencia. Por otro lado, el total final asciende a 221 debido a que algunos de los artículos trabajaban con más de una etapa educativa.

Una vez visto esto, cabe destacar que la educación primaria es la etapa más investigada con un total de 45 documentos. Le sigue la educación secundaria con 33 documentos, y la etapa universitaria con 26; los estudios hacia profesoras y profesores en activo (20); los docentes en formación, así como los estudiantes de bachiller, con sendos 16 documentos; la etapa de educación infantil con 8 documentos; y uno hace referencia a la formación para personas adultas.

Tabla 6

Distribución de la población de referencia

\begin{tabular}{lcc}
\hline Características & Frecuencia & Porcentaje \\
\hline Infantil & 8 & 3,61 \\
Primaria & 45 & 20,36 \\
Secundaria & 33 & 14.93 \\
Bachiller & 16 & 7,23 \\
\hline
\end{tabular}

El pensamiento computacional en educación. Análisis bibliométrico y temático. Roig-Vila, R. y Moreno, V. 


\begin{tabular}{lcc}
\hline Universidad & 26 & 11,76 \\
$\begin{array}{l}\text { Docentes en } \\
\text { formación }\end{array}$ & 16 & 7,23 \\
$\begin{array}{l}\text { Docentes en } \\
\text { activo }\end{array}$ & 20 & 9,04 \\
Formación adulta & 1 & 0,45 \\
No figura & 56 & 25,38 \\
Total & 221 & 100 \\
\hline
\end{tabular}

\section{Lenguajes de programación y plataformas utilizadas}

Se ha realizado una clasificación entre los documentos que recogen de alguna manera qué tipo de lenguaje o plataforma se ha utilizado para trabajar el pensamiento computacional. Tal y como se puede ver en la tabla 7, en 63 de los 189 documentos no se indica este elemento. Esto se encuentra ligado a que 66 de los documentos contienen investigaciones de carácter teórico, donde no es un elemento pertinente el lenguaje o plataforma utilizados.

Por otra parte, en la misma tabla se recogen los lenguajes de programación o instrumentos referidos en los distintos documentos, tanto en investigaciones empíricas como en experiencias educativas. Cabe decir que el número total es superior al número de documentos analizados debido a que algunas de las investigaciones hacían uso de más de un lenguaje o una plataforma de manera simultánea. Así, se recogen en la tabla 7 todos los lenguajes o plataformas que, al menos, se han utilizado en dos ocasiones; y en el apartado "otros", los que solo han aparecido en uno de los documentos analizados.

\section{Tabla 7}

Distribución por lenguajes y plataformas de programación

\begin{tabular}{lcc}
\hline $\begin{array}{l}\text { Lenguajes o plataformas } \\
\text { Utilizados }\end{array}$ & Frecuencia & Porcentaje \\
\hline Figura el lenguaje o plataforma & 126 & 66,67 \\
No figura & 63 & 33,33 \\
Total & 189 & 100 \\
Lenguajes o instrumentos & Frecuencia & Porcentaje \\
utilizados & 52 & 35,13 \\
\hline Diseñado por el autor & 29 & 19,59 \\
Scratch & 14 & 9,46 \\
Robótica & 10 & 6,76 \\
\hline LEGO & & \\
\hline
\end{tabular}

$\overline{\text { El pensamiento computacional en educación. Análisis bibliométrico y temático. Roig-Vila, R. y }}$ Moreno, V. 


\begin{tabular}{lcc}
\hline Programación visual & 5 & 3,38 \\
HTML & 4 & 2,70 \\
C++ & 4 & 2,70 \\
Python & 3 & 2,03 \\
Bebras & 3 & 2,03 \\
Code.org & 3 & 2,03 \\
Alice & 2 & 1,35 \\
Java & 2 & 1,35 \\
Circuitos & 2 & 1,35 \\
Otros & 15 & 10,14 \\
Total & 148 & 100 \\
\hline
\end{tabular}

Como podemos observar en la tabla 7 , vemos que en 52 casos los elementos relacionados con el pensamiento computacional han sido elaborados por la autora o el autor. Esto es debido a que en muchos casos trataban de trabajar aspectos muy concretos, como la abstracción, y eran elaborados ad hoc con dicho fin a través de diferentes códigos que no se mencionan en el documento.

Por otro lado, Scratch es el lenguaje de programación más utilizado, siendo este utilizado en un total de 29 de los documentos. La programación a través de la robótica aparece en 14 documentos, pero se ha distinguido la programación a través de LEGO, de la programación robótica en general, debido a que varios de los artículos lo reflejaban y era un elemento fundamental para la investigación. La programación a través de LEGO es utilizada en 10 de los documentos. El siguiente elemento más mencionado es la programación visual. Se ha etiquetado de esta manera debido a que en algunos de los documentos aparece dicho concepto sin especificar ningún tipo de lenguaje más concreto. Asimismo, podemos observar varios lenguajes de programación como HTML o C++ con una repercusión similar -5 y 4 apariciones respectivamente-

En cuanto a las plataformas que aparecen con más frecuencia, vemos que son Bebras y Code.org., ambas con multitud de recursos educativos relacionados con el pensamiento computacional. Por último, cabe mencionar que en el apartado "otros", que supone un total de 15 elementos, se incluyen lenguajes como Kodu o Logo, entre otros.

\section{DISCUSIÓN Y CONCLUSIONES}

Nos planteábamos como objetivo realizar un estudio bibliográfico sobre el tema del pensamiento computacional y, así, ofrecer una visión a nivel internacional sobre cómo está evolucionando la producción científica de dicho tema en el ámbito educativo. Pues bien, se ha podido comprobar que se trata de un campo de estudio en continuo crecimiento. En los últimos cinco años se ha realizado la gran mayoría de las 
investigaciones en este sentido, lo que nos hace ver, asimismo, lo incipiente del tema. Esto se refuerza con el hecho de que las revistas de divulgación científica especializadas no figuran como grandes productoras en este campo, sino que, en este momento, la fuente principal es el modelo de recopilatorio de actas de eventos científicos. Este hecho puede motivar una predisposición por parte de las revistas especializadas a publicar artículos que aborden este tema, traduciéndose en una buena oportunidad para que se publiquen trabajos que investiguen el pensamiento computacional en el ámbito educativo. Por otro lado, este incremento reciente de publicaciones evidencia la relevancia que está tomando la consideración de la enseñanza de la programación en el aula como una competencia esencial de cara al futuro (Vázquez-Cano y Ferrer, 2015).

Respecto a los objetivos específicos sobre el análisis de los diversos aspectos a estudiar en los respectivos textos, en cuanto a la producción por países, observamos cómo, al margen de Estados Unidos con un total de 66 publicaciones, seguido de China con un total de 36, encontramos a España con 21, a pesar de no estar el pensamiento computacional incorporado dentro en el currículum educativo. Podría suponer ello la posibilidad de ser una oportunidad óptima para poder trabajar de manera coordinada investigadores y agentes educativos. Autores como Valverde, Fernández y Garrido (2015) abogan por incorporar nuevas metodologías y modificar el modelo organizativo de las instituciones educativas con el fin de incluir el pensamiento computacional. Esto constata, pues, el interés del tema reflejado en el número de publicaciones en nuestro país.

Respecto al análisis de producción según las fuentes de publicación, aparecen una gran variedad de fuentes con un número reducido de publicaciones y son más numerosos los recopilatorios de actas de congresos que las revistas. En cuanto a estas, las revistas con más publicaciones sobre pensamiento computacional son Informatics and Education y Red. Revista de Educación a Distancia, con 7 publicaciones cada una. Esto nos permite tener una percepción de lo reciente de este campo de estudio.

Asimismo, otro elemento que refleja claramente el aspecto actual del tema de estudio es la falta de grandes productores, según la clasificación realizada por Crane (1969). Únicamente encontramos 3 autores considerados productores moderados (Song-Charg Kong, Aman Yadav, Uri J. Wilensky). Del mismo modo, predominan los autores clasificados como transeúntes. Esto indica una falta de expertos de referencia en este campo, encontrando únicamente a tres autores que podemos considerar como referentes. Por ello, se da una falta de criterio en el diseño de las investigaciones, así como un inestabilidad y excesiva diversificación de los estudios planteados. Solamente se ha encontrado el hecho de aceptar lo dicho por Wing $(2006,2009)$ como punto de partida para el desarrollo de este concepto. Asimismo, se ha observado un predominio claro de los documentos firmados por más de un autor. De esta manera se siguen los actuales patrones científicos planteados por las tendencias actuales de globalización (Glänzel y Shubert, 2005).

En lo que se refiere a tipos de documentos, aunque los documentos empíricos tienen mayor prevalencia que los teóricos, estos últimos representan una parte destacable. Es una realidad que el simple hecho de alcanzar un consenso sobre qué engloba y representa el pensamiento computacional supone un reto que, hasta ahora, no se ha logrado

El pensamiento computacional en educación. Análisis bibliométrico y temático. Roig-Vila, R. y Moreno, V. 
totalmente. Encontramos diversas tesis como las propuestas por Cearreta-Urbieta (2015), Balladares et al. (2016), Basogain et al. (2015), entre muchos otros, lo cual genera la necesidad de establecer un marco teórico de referencia.

Al mismo tiempo, ha quedado patente que se trata de un concepto que se presta a ser investigado de múltiples maneras. En el análisis se han encontrado estudios que utilizan diversas formas de investigación cuantitativa, así como cualitativa. Aunque la cuantitativa tenga una presencia ligeramente superior, encontramos estudios cualitativos con diversos métodos de recogida de información, permitiendo obtener conclusiones desde diversos enfoques. Igualmente, cabe hacer una valoración, no muy favorable, respecto al hecho de que las investigaciones con un carácter empírico suponen un total de menos de la mitad del total de documentos analizados en el campo de la investigación educativa sobre pensamiento computacional. Esto se contradice con lo indicado por Valverde et al. (2015), quienes defienden que la investigación educativa es la vía para comprender mejor los procesos de enseñanza.

Del mismo modo, vemos cómo cada vez más las investigaciones están enfocadas al desarrollo de instrumentos de evaluación, ya que se trata de un aspecto fundamental del proceso educativo. Encontramos que Grover y Pea (2013) defienden esta tesis, y abogan por orientar parte de la investigación al ámbito de los instrumentos de evaluación.

En relación con la población de referencia a la que va dirigido lo estudiado en los respectivos documentos, cabe destacar que la etapa educativa con mayor número de investigaciones es la educación primaria. Esto resalta la importancia de realizar un acercamiento a las áreas STEM, desde edades tempranas, de modo que más niñas y niños opten por este tipo de carreras profesionales. Como afirma la Unión Europea, dichas áreas están sufriendo un descenso de personas que las estudian, mientras crece su demanda en el ámbito laboral.

Ayudar a integrar el pensamiento computacional desde la primaria supondría un buen punto de partida y así poder desarrollar algunas de las capacidades mencionadas como la abstracción y el pensamiento deductivo. Ahora bien, se presenta una disyuntiva que también debe verse abordada. En la etapa de primaria, únicamente los maestros formados para ello podrían abordar el tema, por lo que se debe hacer un primer planteamiento de cómo formar a los futuros maestros en este campo, aspecto que en la actualidad no está del todo dirimido. En base a ello, concurrimos con lo asegurado por Adell et al. (2019), González et al. (2018), Yadav et al. (2014) y Yadav et al. (2016).

Por último, respecto a los lenguajes de programación y plataformas, a pesar de ser Scratch (Álvarez, 2017) el más utilizado, encontramos que en muchos de los documentos que contenían experiencias empíricas, las investigadoras y los investigadores diseñaban los materiales para llevar a cabo la investigación. En algunos casos, se llega a trabajar incluso con papel y lápiz. Esto demuestra que son diversos los instrumentos que se pueden utilizar para su implementación, lo que sugiere que la falta de alternativas no debería representar un obstáculo a la hora de trabajar el pensamiento computacional. La controversia en este campo no surge por la falta de instrumentos, sino por lo considerado por Zapata-Ros (2015) como principio básico del pensamiento computacional: conocer el mundo de las ideas, sus representaciones y cómo operan.

El pensamiento computacional en educación. Análisis bibliométrico y temático. Roig-Vila, R. y Moreno, V. 
Respecto a las limitaciones del estudio, cabe decir que existen otros indicadores bibliométricos que no se han abordado, pero que podrían considerarse en futuros trabajos. Así, el índice de impacto de los trabajos de los autores o la financiación de los estudios podrían ser aspectos a tener en cuenta. Otra limitación está referida a los textos a los cuales no hemos podido acceder, ni siquiera al resumen, por lo que no se han podido incluir en el estudio.

No obstante, estas limitaciones no impiden, a partir del trabajo realizado, poder afirmar que hay un gran abanico de oportunidades para continuar investigando en esta materia. Se podrían realizar investigaciones más específicas de los distintos aspectos, y así poder ver cómo afecta al desarrollo del pensamiento computacional. Asimismo, se podrían realizar investigaciones de carácter longitudinal, lo que resultaría recomendable para poder comprobar la evolución del alumnado que participe en programas de aprendizaje sobre pensamiento computacional.

De igual forma, encontramos un gran número de opciones de investigación en la distribución de las etapas. Cada etapa requiere unos métodos de enseñanza diferentes y, además, plantear una posible secuenciación de contenidos para la elaboración de futuros currículos que impliquen la inclusión y desarrollo del pensamiento computacional. Por ello, resulta imprescindible que se lleven a cabo investigaciones desde infantil hasta la educación universitaria (García-Peñalvo y Mendes, 2016). Del mismo modo, sería aconsejable que maestras y maestros en activo pudieran participar en investigaciones de estas características. Su conocimiento directo en el entorno y funcionamiento del aula facilitaría en gran medida la realización de dichas investigaciones.

Presentación del artículo: 29 de octubre de 2019

Fecha de aprobación: 27 de febrero de 2019

Fecha de publicación: 31 de mayo de 2020

Roig-Vila, R. y Moreno-Isac, V. (2020). El pensamiento computacional en educación. Análisis bibliométrico y temático. Red. Revista de Educación a Distancia, 20(63). DOI: http://dx.doi.org/10.6018/red.402621

\section{Financiación}

El presente trabajo se enmarca en el seno del Grupo de Investigación "GIDU-EDUTICIN", del Programa de Redes de Investigación en Docencia Universitaria del ICE (REDES-I3CE-2018-4423) y del proyecto Instituto Superior de Investigación Cooperativa IVITRA (Ref.: ISIC/2012/022; http://www.ivitra.ua.es), todos ellos de la Universidad de Alicante.

\section{REFERENCIAS}

Adell, J. S., Llopis, M.A.N., Esteve, M.F.M. y Valdeolivas, N.M.G. (2019). El debate sobre el pensamiento computacional en educación. RIED. Revista Iberoamericana de Educación a Distancia, 22(1), 171-186. doi: http://dx.doi.org/10.5944/ried.22.1.22303

El pensamiento computacional en educación. Análisis bibliométrico y temático. Roig-Vila, R. y Moreno, V. 
Álvarez Rodríguez, M. (2017). Desarrollo del pensamiento computacional en educación primaria: Una experiencia educativa con Scratch. UTE. Revista de Ciències de l'Educació, 1 (2), 45-64. Recuperado de: https://dialnet.unirioja.es/servlet/articulo?codigo $=6229578$

Balladares B, J. A., Avilés S. M. R. y Pérez N. H. O. (2016). Del pensamiento complejo al pensamiento computacional: retos para la educación contemporánea. Sophia, colección de Filosofía de la Educación, 21(1), 143-159.

Basogain, X., Olabe, M. A. y Olabe, J. C. (2015). Pensamiento Computacional a través de la Programación: Paradigma de Aprendizaje. RED. Revista De Educación a Distancia, Número 46. 30 de septiembre de 2015. Recuperado de: https://revistas.um.es/red/article/view/240011

Blikstein, P. (2013). Seymour Papert's legacy: Thinking about learning, and learning about thinking. Seymour Papert Tribute at IDC 2013.

Bocconi, S., Chioccariello, A., Dettori, G., Ferrari, A., Engelhardt, K., Kampylis, P., y Punie, Y. (2016). Exploring the field of computational thinking as a 21 st century skill. Proceedings of the International Conference on Educational and New Learning Technologies July 2016 Barcelona, Spain, 4725-4733.

Bocconi, S., Chioccariello, A., Dettori, G., Ferrari, A., y Engelhardt, K., Kampylis, P., and Punie, Y. (2016). Developing computational thinking in compulsory education. Implications for policy and practice. European Comission, JRC Science for Policy Report.

Brennan, K., y Resnick, M. (2012, April). New frameworks for studying and assessing the development of computational thinking. In AERA 2012 - annual meeting of the American Educational Research Association, Vancouver, Canada (Vol. 1, p. 25). Recuperado de: http://scratched.gse.harvard.edu/ct/files/AERA2012.pdf

Brereton, P., Kitchenham, B.A., Budgen, D., Turner, M., y Khalil, M. (2007). Lessons from applying the systematic literature review process within the software engineering domain. Journal of Systems and Software, 80(4), 571-583. doi: https://doi.org/10.1016/j.jss.2006.07.009

Cearreta-Urbieta, I. (2015). Scratch como recurso didáctico para el desarrollo del Pensamiento Computacional de los alumnos de Secundaria y Bachillerato en la asignatura de Informática y como recurso transversal en el resto de asignaturas. Recuperado de: https://reunir.unir.net/handle/123456789/3150

Centre for Reviews and Dissemination (Ed.) (2009). Systematic Reviews. CRD's guidance for undertaking reviews in health care. York, UK: CRD, University of York. Recuperado de https://goo.gl/731Ehh.

Crane, D. (1969). Social structures in a group of scientist: A test of the "invisible college' hypothesis. American Sociological Review, 34(335), 352.

CSTA y ISTE [International Society for Technology in Education (ISTE) and the Computer Science Teachers Association (CSTA)]. (2011). Operational definition of computational thinking. Recuperado de http://www.iste.org/docs/ctdocuments/computational-thinking-operational-definition-flyer.pdf

Cabrera Delgado, J. M. (2017). Las Ciencias de la Computación en el currículo educativo. Avances en Supervisión Educativa, (27). doi: https://doi.org/10.23824/ase.v0i27.584

Del-Moral Pérez, M., Guzmán Duque, A., y Fernández García, L. (2018). Game-Based Learning: Increasing the Logical-Mathematical, Naturalistic, and Linguistic Learning Levels of Primary School Students. Journal of New Approaches in

El pensamiento computacional en educación. Análisis bibliométrico y temático. Roig-Vila, R. y Moreno, V. 
Educational Research, $\quad$ 3(1), 31-39. doi: http://dx.doi.org/10.7821/naer.2018.1.248

Denning, P. J. (2017). Remaining trouble spots with computational thinking. Communications of the ACM, 60(6), 33-39.

García-Fernández, J. M., Inglés, C. J., Gonzálvez, C., Vicent, M., y Gómez Núñez, M. I. (2013). Familia y discapacidad intelectual: Un estudio bibliométrico de 20002011 a través del social science citation index. Siglo Cero 44 (246), 67-82. Recuperado de: http://hdl.handle.net/10045/43181

García-Fernández, J. M., Inglés, C. J., Vicent, M., Gonzálvez, C., Gómez-Núnez, M. I., y Poveda-Serra, P. (2016). Perfeccionismo durante la infancia y la adolescencia. análisis bibliométrico y temático (2004-2014). Revista Iberoamericana De Psicología Y Salud, 7(2), 79-88. doi: https://doi.org/10.1016/j.rips.2016.02.001

García-López, L., Gutiérrez, D., Pastor, J., y Romo, V. (2018). Validity and Reliability of a Questionnaire on Primary and Secondary School Teachers' Perception of Teaching a Competence-based Curriculum Model. Journal of New Approaches in Educational Research, 7(1), 46-51. doi: http://dx.doi.org/10.7821/naer.2018.1.255

García-Peñalvo, F. J. (2016). What computational thinking is. Journal of Information Technology Research, 9(3), 5-8.

García-Peñalvo, F. J., y Mendes, J. A. (2018). Exploring the computational thinking effects in pre- university education. Computers in Human Behavior, 80, 407-411

Glänzel, W. y Schubert, A. (2005). Analysing scientific networks through co-authorship. En H. F. Moed, W. Glänzel, y U. Sch- moch (Eds.), Handbook of Quantitative Science and Technology Research (pp. 373-388).

González Martinez, J., Estebanell Minguell, M., y Peracaula Bosch, M. (2018). Robotics or Coding? The Concept of Computational Thinking in Pre-service Teachers. Education in the Knowledge Society, 19(2), 29-45.

Grover, S., y Pea, R. (2013). Computational thinking in K-12: A review of the state of the field. Educational Researcher, 42(1), 38-43. Recuperado de: https://journals.sagepub.com/doi/pdf/10.3102/0013189X12463051

Gutiérrez Porlán, I., y Serrano Sánchez, J. (2016). Evaluation and development of digital competence in future primary school teachers at the University of Murcia. Journal of New Approaches in Educational Research, 5(1), 51-56. doi: http://dx.doi.org/10.7821/naer.2016.1.152

Higgins, J.P., y Green, S. (Eds.) (2006). Cochrane handbook for systematic reviews of interventions 4.2.6. The Cochrane collaboration. Recuperado de https://goo.gl/6uU6tM

Ioannou, A., y Makridou, E. (2018). Exploring the potentials of educational robotics in the development of computational thinking: A summary of current research and practical proposal for future work. Education and Information Technologies, 23(6), 2531-2544.

Kitchenham, B. A. (2004). Procedures for performing systematic reviews. Keele University: Technical Report TR/SE-0401 and NICTA Technical Report 0400011T.1. Recuperado de https://goo.gl/wQcMaS

Kong, S. C., Chiu, M. M., y Lai, M. (2018). A study of primary school students' interest, collaboration attitude, and programming empowerment in computational thinking education. Computers \& Education, 127, 178-189.

doi: https://doi.org/10.1016/j.compedu.2018.08.026

El pensamiento computacional en educación. Análisis bibliométrico y temático. Roig-Vila, R. y Moreno, V. 
Moher, D., Liberati, A., Tetzlaff, J., Altman, D.G., \& The PRISMA Group (2009). Preferred reporting items for systematic reviews and meta-analyses: The PRISMA Statement. BMJ, 339, b2535. https://doi.org/10.1136/bmj.b2535

Montero, I., y León, O. G. (2007). A guide for naming research studies in Psychology. International Journal of clinical and Health psychology, 7(3).

Naughton, J. (2012). 'A manifest for teaching computer science in the 21 st century'. The Observer, 31 de marzo.

OCDE. (2018). Job Creation and Local Económic Development 2018. Recuperado de: http://www.oecd.org/cfe/job-creation-and-local-economic-development26174979.htm

Olabe, J. C., Basogain, X., Olabe, M. A., Maíz, I. y Castaño, C. (2014). Solving math and science problems in the real world with a computational mind. Journal of New Approaches in Educational Research, 3(2), 75-82. doi: http://dx.doi.org/10.7821/naer.3.2.75-82

Papert, S. (1980). Mindstorms: Children, computers, and powerful ideas. Basic Books, Inc.

Pérez, N. H. O., y Roig-Vila, R. (2015). Entornos de programación no mediados simbólicamente para el desarrollo del pensamiento computacional. Una experiencia en la formación de profesores de Informática de la Universidad Central del Ecuador. Revista de Educación a Distancia, (46), 1-22. Recuperado de: https://dialnet.unirioja.es/servlet/articulo?codigo $=6022698$

Pinto-Llorente, A. M., Casillas-Martín, S., Cabezas-González, M., y García-Peñalvo, F. J. (2018). Building, coding and programming 3D models via a visual programming environment. Quality \& Quantity, 52(6), 2455-2468. doi: https://doi.org/10.1007/s11135-017-0509-4

Repenning, A., Basawapatna, A. R., y Escherle, N. A. (2017). Principles of Computational Thinking Tools. In P. Rich, y C. Hodges (Eds.), Proceedings of Emerging Research, Practice, and Policy on Computational Thinking (pp. 291305). Recuperado de: https://link.springer.com/chapter/10.1007/978-3-31952691-1_18

Román-González, M. (2015). Test de Pensamiento Computacional: principios de diseño, validación de contenido y análisis de ítems. Perspectivas y avances de la investigación, 279-302.

Sancho Gil, J., y Padilla Petry, P. (2016). Promoting digital competence in secondary education: are schools there? Insights from a case study. Journal of New Approaches in Educational Research, 5(1), 57-63. doi: http://dx.doi.org/10.7821/naer.2016.1.157

Valverde Berrocoso, J., Fernández Sánchez, M. R., y Garrido Arroyo, M. C. (2015). El pensamiento computacional y las nuevas ecologías del aprendizaje. Revista de Educación a Distancia, (46). Recuperado de: https://revistas.um.es/red/article/view/240311

Vázquez-Cano, E. y Ferrer Delgado, D. (2015). La creación de videojuegos con Scratch en Educación Secundaria. Communication papers: media literacy and gender studies, 4(6), 63-73.

Recuperado de: https://dialnet.unirioja.es/servlet/articulo?codigo=5182831

El pensamiento computacional en educación. Análisis bibliométrico y temático. Roig-Vila, R. y Moreno, V. 
Wartenweiler, T. (2018). Serious Play in Education for Social Justice - A Mixed-Methods Evaluation. Journal of New Approaches in Educational Research, 7(1), 61-69. doi: http://dx.doi.org/10.7821/naer.2018.1.260

Wing, J. M. (2006). Computational thinking. Communications of the ACM, 49(3), 33-35.

Wing, J. M. (2009, November). Formal methods for privacy. In International Symposium on Formal Methods (pp. 1-15). Springer, Berlin, Heidelberg.

Yadav, A., Hong, H., y Stephenson, C. (2016). Computational thinking for all: Pedagogical approaches to embedding 21 st century problem solving in $\mathrm{K}-12$ classrooms. Techtrends, 60(6), 565-568. https://doi.org/10.1007/s11528-0160087-7

Yadav, A., Mayfield, C., Zhou, N., Hambrusch, S., y Korb, J. T. (2014). Computational thinking in elementary and secondary teacher education. ACM Transactions on Computing Education, 14(1), 5. doi: 10.1145/2576872

Yagci, M. (2019). A valid and reliable tool for examining computational thinking skills. Education and Information Technologies, 24(1), 929-951. doi: https://doi.org/10.1007/s10639-018-9801-8

Zapata-Ros, M. (2015). Pensamiento computacional: Una nueva alfabetización digital. RED. Revista de Educación a Distancia, (46). Recuperado de: https://www.um.es/ead/red/46/ 